\title{
CONHECIMENTOS DE PAIS DE CRIANÇAS ASMÁTICAS SOBRE A DOENÇA NO MOMENTO DA ADMISSÃO A UM SERVIÇO ESPECIALIZADO
}

\author{
Linjie Zhang*, Marilice G. Costa, laila H. Ávila, Thiago Bonfanti, Emerson H. Ferruzzi
}

Trabalho realizado no Departamento Materno-Infantil, Fundação Universidade do Rio Grande, Rio Grande, RS.

*Correspondência:

Rua Barão de Santa Tecla, 884/202A, Centro, Pelotas, RS, Cep: 96010-140,

Fone: (53) 2258394

zhanglinjie63@yahoo.com.br

\section{RESUMO}

Oвjetivo. Avaliar conhecimentos de pais de crianças asmáticas sobre a doença no momento da admissão a um serviço especializado.

Métodos. 0 presente trabalho foi um estudo descritivo. Foram entrevistados os pais de 93 crianças asmáticas com idade entre 29 dias e 18 anos, no momento da admissão ao ambulatório de Pneumologia Pediátrica de um hospital universitário, por meio de um questionário para avaliar os conhecimentos deles sobre asma. As avaliações concentraram-se no entendimento da natureza, do prognóstico e do tratamento da asma. Foram analisados somente os dados obtidos em pais de 87 crianças com idade entre 29 dias e 10 anos.

REsultados. Na auto-avaliação, 93,1\% dos pais consideraram seus conhecimentos sobre asma insuficientes; e 88,5\% demonstraram interesse em adquirir mais informações. $\mathrm{Na}$ avaliação específica de conhecimentos sobre natureza, prognóstico e tratamento da asma, $96,6 \%$ dos pais não sabiam o papel da inflamação das vias aéreas na síntese dos sintomas da doença; e 5I,7\% acreditavam na cura desta doença; entre os pais cujos filhos usavam nebulizador domiciliar, 80,6\% costumavam cometer erros na sua aplicação; e todos os pais cujos filhos usavam inalador pressurizado sem espaçador não conheciam a técnica inalatória correta; $65,5 \%$ dos pais não tinham consciência da importância sobre o controle do ambiente domiciliar; e a presença de fonte dos alérgenos e de fumantes foi encontrada respectivamente em $77 \%$ e $68,9 \%$ dos domicílios.

ConclusöEs. $O$ presente trabalho mostra a insuficiência de conhecimento sobre asma por parte dos pais de crianças asmáticas no momento da admissão a um serviço especializado, caracterizando-se pelo desconhecimento sobre natureza e prognóstico da doença, técnica inadequada no uso dos fármacos inalatórios e descuido no controle do ambiente domiciliar.

UnITERMos: Asma. Estudo descritivo. Questionário. Conhecimento. Pais.

\section{INTRODUÇÃO}

Asma é a doença crônica mais comum na infância. Nas últimas décadas, apesar dos avanços no entendimento da sua fisiopatogenia e no seu tratamento, a morbi-mortalidade de asma tem crescido em todo o mundo ${ }^{1-4}$. Esta doença em crianças está se tornando um dos principais problemas globais na saúde pública ${ }^{4,5}$.

Devido à cronicidade da doença, o tratamento de asma em crianças é geralmente feito a domicílio, sob a responsabilidade dos pais. Os conhecimentos deles sobre asma podem influenciar na adesão ao tratamento e, conseqüentemente, no controle dos sintomas da doença nos seus filhos ${ }^{4,6}$. A carência de conhecimento sobre asma por parte dos pais de crianças asmáticas foi demonstrada em vários países com níveis socioeconômico e cultural distintos ${ }^{6-10}$. Essa desinformação foi apontada como um dos principais fatores responsáveis pela maior demanda de atendimento em pronto-socorro e maior taxa de hospitalização por crises asmáticas em crianças ${ }^{6,1 !}$. No Brasil, ainda é escassa a avaliação sistematizada do conhecimento sobre asma em pais de crianças asmáticas. Supomos que o difícil acesso ao serviço especializado e a baixa escolaridade dos pais provavelmente predispõem à deficiência de seus conhecimentos sobre asma.

O presente trabalho teve como objetivo avaliar conhecimentos sobre asma dos pais de crianças asmáticas no momento da admissão ao ambulatório de Pneumologia Pediátrica, do Hospital Universitário (HU) da Fundação Universidade do Rio Grande (FURG), da cidade de Rio Grande-RS.

\section{Métodos}

O presente trabalho foi um estudo descritivo. Foram elegíveis para o estudo os pais de todas as crianças (com idade entre 29 dias e 18 anos), com diagnóstico prévio de asma (ou bronquite asmática), no momento da admissão ao ambulatório de Pneumologia Pediátrica do HU da FURG, durante o período de agosto de 1999 a agosto de 2000. O diagnóstico de asma foi confirmado pelo pneumologista no momento da consulta, baseado na história de episódios recorrentes $(>=3$ vezes) de sibilância, com resposta clínica ao uso de broncodilatadores (fenoterol ou salbutamol) isoladamente ou associados com corticóides sistêmicos (prednisona ou prednisolona) $)^{4}$. O HU da FURG é um dos dois hospitais gerais do município de Rio Grande, que tem cerca de 200.000 habitantes. Este hospital possui o único serviço público especializado para 0 atendimento das crianças asmáticas nesta cidade. $O$ número de atendimento no ambulatório de Pneumonlogia Pediátrica foi, em média, de 30 consultas por mês, durante o período do estudo. 
A maior parte do atendimento consistiu em consultas programadas para o acompanhamento das crianças portadoras de doenças respiratórias crônicas, tais como asma, bronquiolopatia pós-viral e displasia broncopulmonar. O projeto da pesquisa foi aprovado pelo Comitê de Ética da FURG e o consentimento escrito foi obtido de todos os participantes.

Após o término do atendimento rotineiro do paciente, um dos pais, dependendo de quem estivesse acompanhando a criança no momento da consulta, foi entrevistado por um dos pesquisadores da equipe, por meio de um questionário (anexo). O questionário foi testado no estudo piloto com 10 pais de crianças asmáticas. As questões concentraram-se no entendimento da natureza, do prognóstico e do tratamento de asma. A avaliação da técnica de nebulização a domicílio foi baseada nas questões $\mid 3$ a $\mid 6$. A técnica foi considerada como inadequada se um dos seguintes fatos costumava ocorrer durante a nebulização: criança dormindo, chupando o bico, colocação inadequada da máscara do nebulizador no rosto do paciente, e volume total do líquido para nebulização menor que $3 \mathrm{ml}$. Na avaliação da técnica do uso de inalador pressurizado, foram incluídos somente os pais das crianças dependentes dos mesmos no uso deste dispositivo. Um inalador placebo (com ou sem espaçador, conforme o uso domiciliar) foi utilizado pelos pais para demonstrar como se aplica este dispositivo em seus filhos. A técnica do uso foi classificada como inadequada se ocorresse qualquer erro na demonstração, conforme as etapas corretas do uso deste dispositivo recomendadas na literatura, ${ }^{4,12}$.

Todos os pesquisadores receberam treinamento adequado para aplicação dos questionários. As questões com palavras de maior complexidade ( $\mathrm{p}$. ex. inflamação, hereditária, contagiosa, tratamento alternativo) foram adaptadas em linguagem ao nível de compreensão dos pais entrevistados e as respostas inadequadas foram corrigidas somente após o término da entrevista.

Os dados provenientes dos questionários foram digitalizados e a análise estatística descritiva foi feita utilizando-se o programa Statistica for Windows 4.3 (Statsoft, Inc. 1993).

\section{Resultados}

Um total de 93 crianças asmáticas foram atendidas pela primeira vez no ambulatório de Pneumologia Pediátrica do HU da FURG durante o período do estudo. Todos os pais das 93 crianças asmáticas participaram da pesquisa, sendo que $90(96,8 \%)$ eram mães. Considerando-se a grande amplitude na faixa etária das crianças incluídas, foram analisados somente os dados obtidos em pais de 87 crianças com idade entre 29 dias e 10 anos, excluindo adolescentes. As características pessoais e familiares das 87 crianças asmáticas são mostradas na Tabela I.

A fonte principal de conhecimentos sobre asma foi o médico $(80,8 \%)$, mas $61,6 \%$ dos pais queixaram-se de ter tido informações insuficientes. A Tabela 2 mostra os principais resultados da avaliação dos conhecimentos sobre asma em pais de crianças asmáticas. Quanto ao entendimento da natureza e do prognóstico de asma, $96,6 \%$ dos pais não sabiam o papel de inflamação das vias aéreas na síntese dos sintomas da doença e a metade acreditava na cura de mesma.

Nas crises de asma, $75,9 \%$ dos pais usavam beta- 2 agonistas antes

\begin{tabular}{|c|c|}
\hline Características & $\mathbf{N}^{0}$ de pacientes (\%) \\
\hline Sexo(masculino) & $42(48,3)$ \\
\hline Idade (anos, média $\pm D P$ ) & $4,4 \pm 3,3$ \\
\hline Tempodedoença (anos, média $\pm D P$ ) & $3,2 \pm 2,8$ \\
\hline Hospitalizaçãonosúltimos 12 meses & $42(48,3)$ \\
\hline Rendafamiliar per capita (< I salário mínimo/mês) & $66(75,9)$ \\
\hline Aglomeracão (> 2pessoas/peça) & $17(19,5)$ \\
\hline \multicolumn{2}{|l|}{ Escolaridade dos pais (< 8 anos) } \\
\hline $\mathrm{Pai}$ & $61(70,1)$ \\
\hline Mãe & $51(58,6)$ \\
\hline Históriafamiliardeatopia & $58(66,7)$ \\
\hline
\end{tabular}

Tabela 2 - Avaliação de conhecimento sobre asma em 87 pais de crianças asmáticas

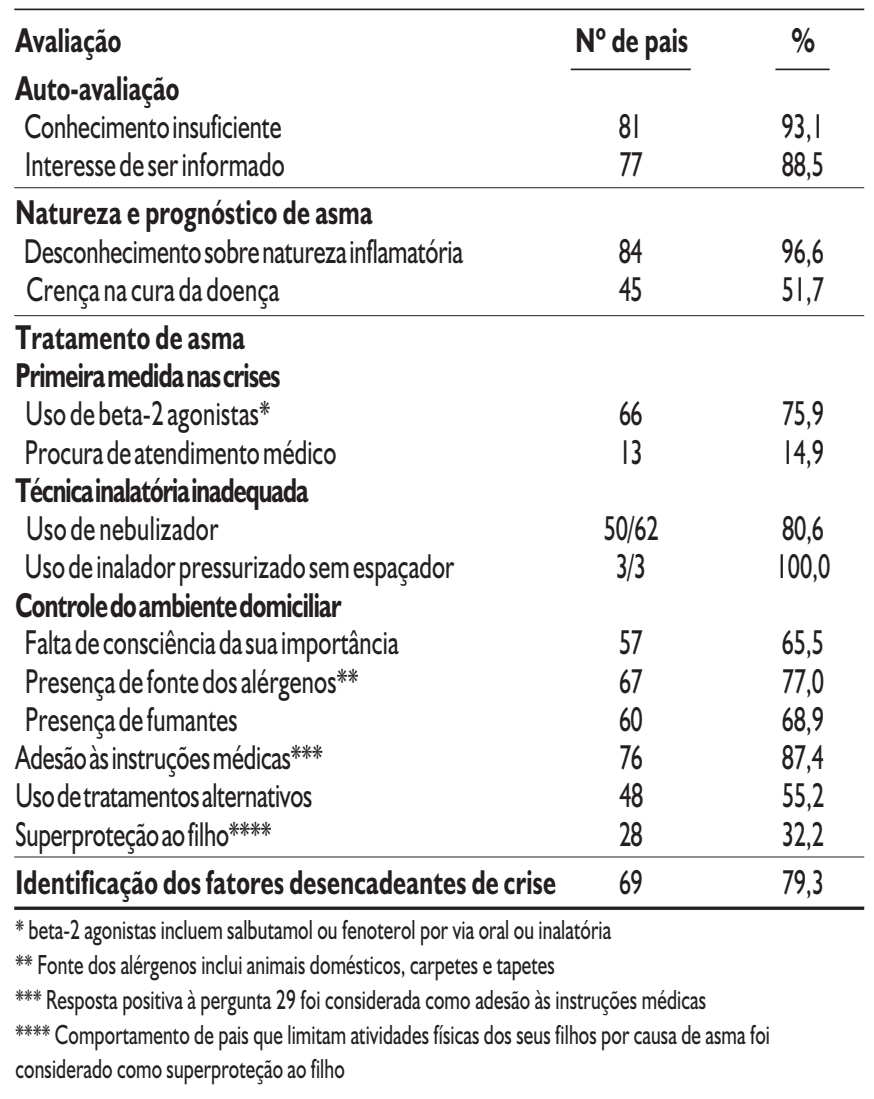

de procurar atendimento médico. Sessenta e duas $(71,3 \%)$ famílias tinham nebulizador no domicílio, mas $80,6 \%$ dos pais não dominavam a técnica correta de fazer a nebulização. $O$ erro mais freqüente foi a realização de nebulização durante o sono (62,9\%), seguindo-se de colocação inadequada da máscara do nebulizador no rosto do paciente $(54,8 \%)$. Apenas seis $(6,9 \%)$ pacientes usavam inalador pressurizado para administrar broncodilatadores, dos quais cinco eram dependentes dos seus pais para o uso deste dispositivo. Dois pais cujos filhos usavam inalador com espaçador, mostraram técnica adequada do uso; porém, os três pais cujos filhos usavam inalador sem espaçador não conheciam 
as etapas corretas do uso deste dispositivo. Na demonstração, os três pais dispararam, simplesmente, o inalador dentro da boca dos seus filhos, sem nenhuma orientação sobre manobras respiratórias antes e depois do disparo. Quanto ao controle do ambiente domiciliar, 65,5\% dos pais não tinham consciência da sua importância; e a presença dos fumantes e da fonte dos alérgenos (animais domésticos, carpetes e tapetes) no domicílio foi freqüente.

Sessenta e nove pais $(79,3 \%)$ observaram alguns fatores desencadeantes de crises de asma em seus filhos, dentre os quais a mudança climática, o resfriado e a fumaça de cigarro foram os mais freqüentes.

\section{Discussão}

Asma é uma doença inflamatória crônica das vias aéreas, ainda considerada incurável, mas controlável $l^{4}$. O presente trabalho mostra que $96,6 \%$ dos pais não conheciam o papel da inflamação das vias aéreas na síntese dos sintomas de asma; e $51,7 \%$ acreditavam na cura da doença. Essa desinformação sobre natureza e prognóstico de asma pode dificultar a compreensão da cronicidade da doença e da necessidade do tratamento regular e prolongado em muitos pacientes. Apesar de a maioria dos pais $(87,4 \%)$ declararem adesão às instruções médicas, o uso de tratamentos alternativos foi relativamente freqüente $(55,2 \%)$ neste grupo de pacientes e esses dados refletem, provavelmente, o descontentamento dos pais quanto aos resultados dos tratamentos convencionais de asma.

Os medicamentos devem ser, preferencialmente, administrados aos asmáticos por via inalatória ${ }^{4,5}$. 0 dispositivo mais recomendado para inaloterapia em crianças é o inalador pressurizado (bombinha) acoplado a um espaçador adequado $0^{4,5,13}$. Este trabalho mostra que 0 nebulizador ainda é mais freqüentemente indicado do que o inalador pressurizado para administrar broncodilatadores $(73,1 \%$ vs $6,9 \%)$ pelos médicos não especialistas em crianças asmáticas. Agrande preocupação em relação ao uso de nebulização éa qualidade do nebulizador domiciliar que, muitas vezes, não é adequada para fornecer aerossol inalável, devido ao baixo fluxo gerado pelo compressor elétrico ${ }^{4}$. A vantagem da nebulização é a técnica de inalação mais fácil e isso pode levar os médicos não especialistas a indicar este dispositivo com maior freqüência. Apesar da facilidade técnica do uso, $80,6 \%$ dos pais costumavam cometer alguns erros na realização de nebulização a domićílio. Os erros mais freqüentes foram a aplicação de nebulização durante o sono e a colocação inadequada da máscara no rosto do paciente. Esses erros técnicos podem causar maior perda de medicamentos e maior depósito dos mesmos na orofaringe e, conseqüentemente, redução da efetividade do tratamento e aumento dos efeitos indesejáveis ${ }^{12}$.

O inalador pressurizado é o dispositivo com eficácia mais unifor$\mathrm{me}^{4,13}$. O uso de um espaçador adequado, além de reduzir o depósito de medicamentos na orofaringe e aumentar o depósito pulmonar, pode tornar a técnica de inalação mais fácil ${ }^{4,12}$. No presente trabalho, o pequeno número dos pacientes que usavam inalador pressurizado com espaçador não permite uma conclusão precisa sobre o nível de conhecimento dos pais quanto ao uso deste dispositivo, mas dois pais cujos filhos usavam este aparelho demonstraram técnica adequada do uso. Quanto ao inalador pressurizado sem espaçador, a técnica inalatória é mais difícil, devido à necessidade de coordenação entre disparo e respiração. O presente trabalho mostra que nenhum dos três pais cujos filhos usavam inalador pressurizado sem espaçador dominou a técnica correta do uso. Esses dados indicam a necessidade de espaçador para o uso de inalador pressurizado em crianças.

O controle ambiental deve fazer parte do tratamento dos pacientes asmáticos ${ }^{4,5}$. Além da poeira domiciliar, destaca-se a fumaça de cigarro no domicílio como um fator ambiental importante, sobre o qual existem estudos comprovando seu malefício em crianças asmáticas ${ }^{14,15}$. O presente trabalho mostra que $68,9 \%$ dos pacientes tinham pelo menos um dos pais fumantes dentro de casa. A presença de fonte dos alérgenos no domicílio também foi freqüente. A maioria dos pais $(65,5 \%)$ não tinha consciência da importância do controle ambiental e isso pode ser um dos fatores responsáveis por essa elevada prevalência do descuido no controle do ambiente domiciliar.

A principal fonte de conhecimentos dos pais sobre asma foram os médicos, mas a maioria $(61,6 \%)$ desses pais considerou insuficientes as informações recebidas. Este resultado mostra que a comunicação entre os médicos e os pais das crianças asmáticas é inadequada, sendo, provavelmente, responsável pela falta de conhecimentos destes sobre asma.

As limitações metodológicas deste trabalho devem ser consideradas na interpretação dos resultados. O delineamento de estudo descritivo não permite a identificação dos fatores associados ao conhecimento dos pais sobre asma, como tempo de doença, idade e escolaridade dos pais e tipo de atendimento médico prévio. O questionário utilizado neste trabalho foi testado no estudo piloto, mas sua validade não foi avaliada. Como o questionário contém somente as questões essenciais sobre asma e está em linguagem de fácil compreensão com exceção de algumas palavras, acreditamos que o mesmo pode ser considerado como um instrumento adequado para a proposta do estudo.

\section{Conclusão}

Em resumo, o presente trabalho mostra a insuficiência de conhecimentos sobre asma por parte dos pais de crianças asmáticas no momento da admissão ao ambulatório de Pneumologia Pediátrica do HU da FURG, caracterizando-se pelo desconhecimento sobre natureza e prognóstico da doença, técnica inadequada no uso dos fármacos inalatórios e descuido no controle do ambiente domiciliar. Esses resultados reforçam a necessidade de educação dos pais de crianças asmáticas.

Conflito de interesse: não há.

\section{SUMMARY}

Asthma related knowledge among parents of asthmatic CHILDREN AT THE MOMENT OF ADMISSION TO A SPECIALIZED SERVICE

OBJECTIVE. To assess asthma related knowledge among parents of asthmatic children at the moment of admission to a specialized service.

Methods. This was a descriptive study. Parents of 93 asthmatic children (aged from 29-18 yr) were interviewed, at the moment of admission to the Pediatric Pulmonary Outpatient Service of a university hospital, using a questionnaire to assess their asthma related knowledge. 
The assessment focused on understanding of the nature, prognosis and treatment of asthma. Only the data from parents of 87 children aged from 29 days to 10 years were analyzed.

RESULTS. Upon self-assessment, 93.1\% of the parents considered their asthma related knowledge insufficient and $88.5 \%$ showed interest in obtaining more information. With regard to understanding of the nature, prognosis and treatment of asthma, $96.6 \%$ of the parents did not know the role of airway inflammation on synthesis of symptoms and $51.7 \%$ believed in a cure for the disease. Among the parents whose children use a home nebulizer, $80.6 \%$ used it incorrectly; all of the parents whose children use spray without a spacer were not familiar with the correct inhalation technique; $65.5 \%$ of the parents were unaware of the importance of environmental control. Presence of allergic sources and of smokers was found in $77.0 \%$ and $68.9 \%$ of families respectively.

Conclusions. This study shows an insufficient asthma related knowledge among parents of asthmatic children at the moment of admission to a specialized service, characterized by ignorance about the nature and prognosis of the disease, inadequate technique to use inhaled drugs and unawareness of home environmental control. [Rev Assoc Med $2005 ; 51(6): 342-7]$

KEY WORDS: Asthma. Descriptive study. Questionnaire. Knowledge. Parent.

\section{RefERÊNCIAS}

I. Akinbami LJ, Schoendorf KC. Trends in childhood asthma: prevalence, health care utilization, and mortality. Pediatrics 2002; I I 0(2 pt I ):3 I 5-22.

2. Mitchell EA. International trends in hospital admission rates for asthma. Arch Dis Child I 985;60:376-9.

3. Chatkin JM, Barreto SM, Fonseca NA, Gutierrez CA, Sears MR. Trends in asthma mortality in young people in souther Brazil. Ann Allergy Asthma Immunol I999; 82:287-92.
4. Sociedade Brasileira de Alergia e Imunopatologia, Pediatria, Pneumologia e Tisiologia. II Consenso Brasileiro no manejo da asma. J Pneumol 1998;24:173-276.

5. National Institute of Health. National Heart, Lung and Blood Institute. Global initiative for asthma (GINA). Bethesda: NHLBI/WHO workshop; 1995. (Publication Number 95-3659).

6. Van Asperen P, Jandera E, De NeefJ, Hill P, Law N. Education in childhood asthma: a preliminary study of need and efficacy. Aust Pediatr J 1986;22:49-52.

7. Bernard-Bonnin AC, Pelletier H, Allard-Dansereau C, Robert M, Masson $P$, Matheux B, et al. Parental knowledge about their asthmatic children. Pediatrie 1991;46:489-97.

8. Lal A, Kumar L, Molhotra S. Knowledge of asthma among parents of asthmatic children. Indian Pediatrics 1995;32:649-55.

9. Jones SL, Weinberg M, Ehrlich RI, Roberts K. Knowledge, attitudes, and practices of parents of asthmatic children in Cape Town. J Asthma 2000;37:519-28.

10. Fadzil A, Norzila MZ. Parental asthma knowledge. Med J Malaysia 2002; 57: 474-8I.

II. Conway SP, Littlewood JM. Admission to hospital with asthma. Arch Dis Child I 985;60:636-9.

12. Laube BL. Aerosol delivery systems. In: Loughlin GM, Eigen H, editors. Respiratory disease in children: diagnosis and management. Baltimore: Williams \& Wilkins; 1994. p.721-9.

13. British Thoracic Society, National Asthma Campaign, Royal College of Physician of London, et al. British guidelines on asthma management 1995 review and position statement. Thorax 1997;52(Suppl I):s2-s 10.

14. Murray AB, Morrison BJ. The effect of cigarette smoke from the mother on bronchial responsiveness and severity of symptoms in children with asthma. J Allergy Clin Immunol I 986;76:575-8I .

15. Landau LI. Parental smoking: asthma and wheezing illness in infants and children. Paediatr Respir Rev 200 I;2:202-6.

Artigo recebido: 05/07/04 Aceito para publicação: 02/02/05 


\section{ANEXO: QUESTIONÁRIO DE AVALIAÇÃO DO CONHECIMENTO SOBRE ASMA DE PAIS COM CRIANÇAS ASMÁTICAS}

\section{IDENTIFICAÇÃO DO PACIENTE}

Nome: Idade(meses): Sexo: $M() F()$

$N^{0}$ registro:

Endereço:

PESSOAENTREVISTADA Pai ( ) Mãe ( ) Outras( )

\section{PARTE I. INFORMAÇÕES GERAIS}

\section{Perfil socioeconômico da família}

Númerodemembros dafamilia:

Rendafamiliar(saláriosmínimos/mês):

Habitação (número de peças):

Grau de instrução dos pais(anos): Pai Mãe

Idadedos pais(anos):

Pai Mãe

Profissão dos pais:

Pai

Mãe

\section{História familiar de atopia}

Asma:

Rinitealérgica:

Dermatiteatópica:

Alergiaalimentar:

\begin{abstract}
Não ( )
\end{abstract}
Não ( )

Não( )

Não ( )

\section{$\operatorname{Sim}(\quad)$}

$\operatorname{Sim}(\quad)$

$\operatorname{Sim}()$

$\operatorname{Sim}()$

Quem:

Quem:

Quem:

Quem:

Idade no início da doença (meses):

Freqüência de internações nos últimos 12 meses:

\section{PARTE II. AVALIAÇÃO DE CONHECIMENTO SOBRE ASMA}

\section{Auto-avaliação do nível de conhecimento sobre asma}

I. Você considera o nível do seu conhecimento sobre asma como: Suficiente ( ) Insuficiente ( )

2. De onde vêm seus conhecimentos sobre asma: Médico（） Livro（） Amigos（） Outros（）

3. Você está satisfeito com informações fornecidas pelo médicos que atenderam seu (sua) filho(a): $\operatorname{Sim}($ ) Não( )

4. Você gostaria de ter mais informações sobre asma: $\operatorname{Sim}($ ) Não( )

\section{Conhecimento sobre natureza e prognóstico de asma}

5. Você acha que asma é uma doença hereditária: $\operatorname{Sim}($ ) Não（） Sem opinião（）

6. Você acha que asma é uma doença contagiosa: $\operatorname{Sim}($ ) Não（） Sem opinião（）

7. Você sabe que os brônquios dos asmáticos estão inflamados e isso pode causar tosse, chiado no peito e falta de ar: Sim（） Não( ) Sem opinião( )

8. Você acha que asma tem cura: $\operatorname{Sim}(\quad)$ Não（） Sem opinião（）

9. Você acha que os sintomas de asma podem ser controlados pelo tratamento: $\operatorname{Sim}($ ) Não( ) Sem opinião( )

Identificação dos fatores desencadeantes de crises asmáticas

10. Quais das seguintes causas podem provocar crises de asma em seu(sua) filho(a):

Mudança de temperatura ( ) Resfriado( ) Fumaça de cigarro( ) Poeira( ) Exercício físico( ) Pêlos de animais( ) Alimentos( )

Cheiroforte( ) Outras( ) Nenhum( ) Sem opinião( )

Conhecimento sobre tratamento de asma

II. Qual a primeira medida quevocê costuma tomar quandoseu(sua)filho(a) estácom crise deasma: Nebulização com berotec ou aerolin( ) Bombinha de berotec ou aerolin( ) Xarope de berotec ou aerolin( ) Chá caseira ( ) Consulta médica ( ) 


\section{ANEXO: QUESTIONÁRIO DE AVALIAÇÃO DO CONHECIMENTO SOBRE ASMA DE PAIS COM CRIANÇAS ASMÁTICAS (CONTINUAÇÃO)}

12. Você tem nebulizador em casa: $\operatorname{Sim}($ ) Não( )

Se sim, vá para a pergunta 13; se não, vá para a pergunta 18

13. Você costuma fazer a nebulização quando seu(sua) filho(a) está dormindo: $\operatorname{Sim}($ ) Não( )

14. Você costuma fazer a nebulização quando seu(sua) filho(a) está chupando o bico: $\operatorname{Sim}($ ) Não( )

15. Você costuma encostar a máscara do nebulizador firmemente no rosto do seu(sua) filho(a) quando faz a nebulização: Sim( ) Não( )

16. Quantos mililitros desorofisiológico vocêcostuma colocar parafazer uma nebulização:

17. Você limpa o nebulizador regularmente: Não( ) Sim( ) Qual a freqüência?

18. Seu(sua) filho(a) usa a bombinha de berotec ou aerolin: Não( ) $\operatorname{Sim}($ )

Se sim, vá para a pergunta 19; se não, vá para a pergunta 21

19.Seu(sua) filho(a) usa a bombinha com o espaçador: $\operatorname{Sim}($ ) Não( )

20.Seu(sua) filho(a) usa a bombinha sempre com sua ajuda: $\operatorname{Sim}($ ) Não( )

Demonstração do uso da bombinha se a resposta à pergunta 20 for sim

21. Você acha que o uso de berotec ou aerolin na dose recomendada pelo médico pode causar alguns problemas à saúde do seu(sua) filho(a): Não( ) Sem opinião( ) Sim( ) Quais:

22. Você acha que o controle do ambiente domiciliar é importante para diminuir os sintomas de asma do seu(sua) filho(a): Sim( ) Não( ) Sem opinião( )

23. Tem alguém que fuma dentro de sua casa: Não（） Sim（）Quem e quantos cigarros/dia?

24. Tem alguns animais domésticos dentro de sua casa: Não( ) $\operatorname{Sim}($ ) Quais:

25. Tem tapetes ou carpetes dentro de sua casa: Não（） Sim（ ) Quais:

26. Com que freqüência você lava a roupa de cama:

27. Com que freqüênciavocêlimpa sua casa:

28. Você limita atividades do seu(sua) filho(a) (p. ex. brincar com outras crianças, tomar banho na piscina ou praia, viajar, ir para festa, etc.) por medo de causar a crise de asma:

$\operatorname{Sim}($ ) Não( )

29. Você segue exatamente as orientações do médico do seu(sua) filho(a) sobre o tratamento: Sim( ) Não( )

30.Seu(sua) filho(a) recebeu algum tipo de tratamento alternativo (acupuntura, ervas medicinais, homeopatias, outros) para asma: Não( ) Sim( )

Quais: 